\title{
Detection of intrinsic variability in the eclipsing massive main-sequence O+B binary HD 165246
}

\author{
C. Johnston, ${ }^{1 \star}$ B. Buysschaert, ${ }^{1,2}$ A. Tkachenko, ${ }^{1 \star}$ C. Aerts ${ }^{1,3}$ and C. Neiner ${ }^{2}$ \\ ${ }^{1}$ Instituut voor Sterrenkunde, KU Leuven, Celestijnenlaan 200D, 3001 Leuven, Belgium \\ ${ }^{2}$ LESIA, Observatoire de Paris, PSL Research University, CNRS, Sorbonne Universités, UPMC Univ. Paris 6, Univ. Paris Diderot, Sorbonne Paris Cité, 5 \\ Place Jules Janssen, F-92195 Meudon, France \\ ${ }^{3}$ Department of Astrophysics, IMAPP, Radboud University Nijmegen, NL-6500 GL, Nijmegen, the Netherlands
}

Accepted 2017 April 21. Received 2017 April 21; in original form 2017 March 13

\begin{abstract}
We present the analysis of $29.77 \mathrm{~d}$ of $K 2$ space photometry of the well-detached massive 4.6 d O+B binary HD 165246 ( $V=7.6)$ obtained during Campaign 9b. This analysis reveals intrinsic variability in the residual light curve after subtraction of the binary model, in the frequency range $[0,10] \mathrm{d}^{-1}$. This makes HD 165246 only the second $\mathrm{O}+\mathrm{B}$ eclipsing binary with asteroseismic potential. While some of the frequencies are connected with the rotation of the primary, others are interpreted as due to oscillations with periodicities of order days. The frequency resolution of the current data set does not allow us to distinguish between frequencies due to standing coherent oscillation modes or travelling waves. Future timeresolved high-precision spectroscopy covering several binary orbits will reveal whether HD 165246 is a Rosetta stone for synergistic binary and seismic modelling of an O-type star.
\end{abstract}

Key words: asteroseismology - techniques: photometric - binaries: eclipsing-stars: individual: HD 165246 - stars: massive - stars: variables: general.

\section{INTRODUCTION}

The past decade has brought revolutionary advances in the fields of asteroseismology and stellar evolution for intermediate- to low-mass main-sequence (MS) and red giant stars given the high-precision, high duty-cycle light curves assembled by the spacebased COROT (Auvergne et al. 2009) and nominal Kepler missions (Koch et al. 2010). Some of the most significant advances for intermediate- (A-F) to high-mass (O-B) MS stars include (i) determination of surface-to-core rotation profiles (Kurtz et al. 2014; Saio et al. 2015; Murphy et al. 2016; Schmid \& Aerts 2016), (ii) identification of regular g-mode period series and their use to constrain rotation profiles (Van Reeth et al. 2015; Pápics et al. 2017; Triana et al. 2015) and (iii) calibration of chemical mixing caused by rotation and overshooting (Moravveji et al. 2016; Van Reeth, Tkachenko \& Aerts 2016). In addition, Kepler delivered a few thousand eclipsing binaries, which allow for largely model-independent estimates of stellar parameters. The co-evol nature of binaries provides the only other calibration for internal physics, such as mixing and overshooting, by forcing model evaluations at the same ages against spectroscopy, as was shown by Claret \& Torres (2016). In the cases where pulsations are observed in eclipsing binaries, asteroseismic and binary modelling produces complementary parameter estimates, enabling synergistic modelling from two independent

^E-mail: colecampbell.johnston@kuleuven.be (CJ); andrew.tkachenko@ ster.kuleuven.be (AT) methods. These systems are of high astrophysical importance due to their unique ability to constrain internal physics to a high precision. In unique cases, binary systems with a high eccentricity have been shown to exhibit periastron brightening and tidally excited pulsations (e.g. Welsh et al. 2011; Hambleton et al. 2016). Pablo et al. (2017) recently reported the first case of an eccentric O-type binary exhibiting tidally induced pulsations with the BRITE-constellation satellites, although the system does not show eclipses.

Due to a scarcity of O-type stars in the COROT fields, and an altogether lack thereof in the nominal Kepler field, these stars have not been analysed in such detail as their lower mass counterparts. This has left models of these stars' interiors uncalibrated by asteroseismology or high-precision binary modelling and hence has not allowed to evaluate physical mechanisms such as rotational mixing and core overshooting in massive stars. On the other hand, internal gravity waves (IGWs) generated at convective/radiative boundaries inside massive stars that transport chemicals and angular momentum (e.g. Rogers et al. 2013; Alvan et al. 2015) have recently been detected in high-precision space data (Aerts \& Rogers 2015; Aerts et al. 2017). However, while seismic analysis of coherent oscillation modes compares theoretically computed frequencies for a given stellar model against observationally determined frequencies, no direct method of comparison has yet been developed for analysis of IGWs. Instead, observations of IGWs are compared with computationally expensive 2D and 3D simulations that require a fixed mass and age ranges as input.

After its re-purposing, the $K 2$ mission began observing multiple fields, providing the opportunity for high-quality space-based 
observations of massive stars, such as HD 165246. Originally characterized as an O8 V star by Walborn (1972), HD 165246 (EPIC 224466741) was later discovered to be an eclipsing binary by Otero (2007), making this object a prime candidate for binary modelling to constrain interior physics. Mayer, Harmanec \& Pavlovski (2013) assembled spectroscopy alongside 617 photometric ASAS observations taken in the $V$-band to model the binarity of the system with the PHOEBE binary modelling code (Prša \& Zwitter 2005). Their analysis rendered a complete orbital solution with a $4.6 \mathrm{~d}$ period assuming no third light, revealing a slight eccentricity, supersynchronous rotation in the hot primary and an argument of periastron near $90^{\circ}$. However, the authors noted caution at the value of the eccentricity and suggested that it should be investigated further with higher quality data.

Sana et al. (2014) utilized interferometric observations obtained with the Sparse Aperture Masking mode of the NACO instrument on the Very Large Telescope at ESO to deduce that the $4.6 \mathrm{~d}$ binary studied by Mayer et al. (2013), denoted as HD 165246Aa+Ab, is a member of a sextuple system with the companions being 30 mas (HD 165246B), $1.9 \operatorname{arcsec}$ (HD 165246C), $6.6 \operatorname{arcsec}$ (HD 165246D) and 7.9 arcsec (HD 165246E) away. Furthermore, Sana et al. (2014) reported magnitude contrasts in the $H$ band, revealing that the closest two members to the inner binary $(\mathrm{B}+\mathrm{C})$ could provide anywhere from 7.2 to 43.35 per cent (within $1 \sigma$ ) of the total light observed from the system, with the large uncertainties arising from the tertiary being located at the detector limit of the NACO instrument.

In the current analysis, we report the detection of intrinsic variability in the $K 2$ light curve of HD 165246, implying that this object may become a Rosetta stone for future asteroseismic calibration of the class of massive MS O-type stars. Furthermore, the detection of this eclipsing system provides different constraints than the $\iota$ Ori system (Pablo et al. 2017) which uses the periastron brightening event to determine the system inclination.

\section{K2 PHOTOMETRY}

HD 165246 was observed in both the long-cadence (LC; $29.42 \mathrm{~min}$ integration time) and short-cadence (SC; $58.85 \mathrm{~s}$ integration time) modes from 2016 April 21 to July 1 by the re-purposed $K 2$ space satellite as a part of proposal GO9913. K2 (Howell et al. 2014) is the continuation of the nominal Kepler mission that observed a single field between Cygnus and Lyrae for nearly $4 \mathrm{yr}$ with $\mu$ mag precision before the loss of two reaction wheels. Although $K 2$ suffers from a loss in precision and observational time base, it observes more fields, including those containing massive stars.

Community efforts to reduce $K 2$ data have been made, resulting in a host page at the Mikulski Archive for Space Telescope ${ }^{1}$ for the data extracted according to Vanderburg \& Johnson (2014). Inspection of the target pixel masks reduced according to Vanderburg \& Johnson (2014) reveals that multiple contaminating sources were included in the original mask of HD 165246 (Fig. 1). To minimize contamination, we downloaded the original target pixel data and performed a custom reduction according to Buysschaert et al. (2015), avoiding the contaminating sources included in the original mask. A comparison of the two masks can be seen in Fig. 1 and illustrates why our reduction is more appropriate to study the binary itself. It is important to note that all six members of the sextuple system are covered by just two of the large $3.96 \times 3.96$ arcsec

\footnotetext{
${ }^{1}$ MAST; https://archive.stsci.edu/k2/
}

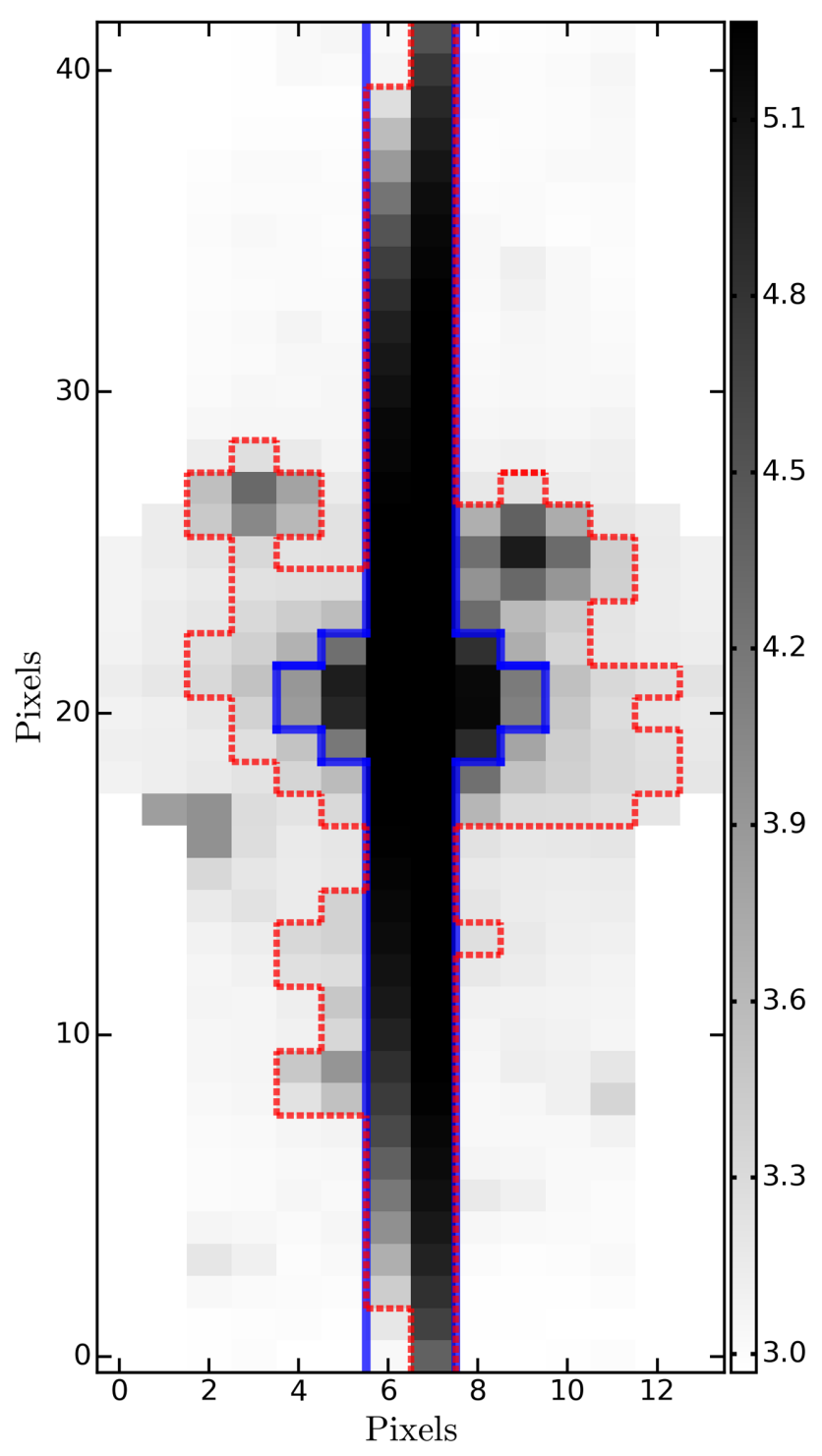

Figure 1. Comparison of the original mask (red-dashed) and our custom mask (blue) used to extract the $K 2$ light curve. The grey-scale indicates the logarithm of the co-added flux of all images (in e/s). Pixels are $3.96 \times 3.96$ arcsec each.

Kepler pixels. Our reduction resulted in nearly $30 \mathrm{~d}$ of high-quality science data for which instrumental effects are negligible (some 1400 data points). As this is a bright target $(V=7.6)$, it saturates the Kepler pixels, smearing the signal across several of them. This allows us to check that the binary signal and other variability originate from the same source by varying the pixel mask and comparing the resulting light curves. As such, we find that the variability remains in the light curve despite quite drastic variations in mask selection for data extraction, always avoiding the contaminating sources.

\section{LIGHT-CURVE ANALYSIS}

Initial inspection of the light curve reveals a moderate reflection effect and non-sphericity of the primary given the increasing flux towards secondary eclipse, flat bottom eclipses and apparent variable signal present in both primary and secondary eclipses, suggesting an intrinsically variable primary. Meaningful investigation of the variability requires a two-step light-curve modelling approach by 
Table 1. Varied and derived parameters for PHOEBE modelling, adopting the parameter definitions from Prša \& Zwitter (2005). BJD $_{0}$ refers to a previous time of superior conjunction.

\begin{tabular}{|c|c|c|}
\hline Parameter & Prior range & Estimate \\
\hline$P_{\text {orb }}(\mathrm{d})$ & $(4.5920,4.5930)$ & $4.592834 \pm 3 \times 10^{-6}$ \\
\hline $\mathrm{BKJD}_{0}(\mathrm{JD}-2454833)$ & $(2382.39,2382.418)$ & $2382.4002 \pm 5.7 \times 10^{-5}$ \\
\hline $\mathrm{q}$ & $(0.05,0.3)$ & $0.1723 \pm 0.0004$ \\
\hline$i(\mathrm{deg})$ & $(80,90)$ & $83.413 \pm 0.006$ \\
\hline $\operatorname{sma}\left(\mathrm{R}_{\odot}\right)$ & $(32,36)$ & $34 \pm 2$ \\
\hline$e$ & & 0 (fixed) \\
\hline$L_{1}$ & $(9.5,12)$. & $10.230 \pm 0.002$ \\
\hline$L_{2}$ & $(0.01,1)$. & $0.223 \pm 0.002$ \\
\hline$L_{3}$ (per cent) & $(0.01,0.5)$ & $0.1801 \pm 0.0002$ \\
\hline $\operatorname{Potential}_{1}\left(\Omega_{1}\right)$ & $(4.5,10.0)$ & $5.1006 \pm 0.0005$ \\
\hline Potential $_{2}\left(\Omega_{2}\right)$ & $(3.5,10.0)$ & $3.991 \pm 0.006$ \\
\hline$T_{\mathrm{eff}, 1}(\mathrm{~K})$ & & 33000 (fixed) \\
\hline$T_{\mathrm{eff}, 2}(\mathrm{~K})$ & $(10000,17000)$ & $12600 \pm 150$ \\
\hline Albedo $_{1}\left(A_{1}\right)$ & & 1 (fixed) \\
\hline Albedo $_{2}\left(A_{2}\right)$ & $(0,1)$ & 1 (fixed) \\
\hline$f_{2}\left(\frac{P_{\mathrm{rot}}}{P_{\mathrm{orb}}}\right)$ & $(0.1,3)$. & $1.37 \pm 0.101$ \\
\hline$R_{1}\left(\mathrm{R}_{\odot}\right)$ & & $7.12 \pm 0.34$ \\
\hline$R_{2}\left(\mathrm{R}_{\odot}\right)$ & & $2.30 \pm 0.12$ \\
\hline
\end{tabular}

Note. $L_{1}, L_{2}$ and $L_{3}$ are scaled passband luminosities within PHOEBE.

which we first fit the binary orbit, remove it and then examine the residuals via time-series analysis.

\subsection{Binary modelling}

Utilizing the re-extracted $K 2$ data, we improve upon the initial binary solution by Mayer et al. (2013). Based on their assembled spectroscopy, we fix the effective temperature and projected rotational velocity of the primary in our analysis. We model the light curve with the emcee python module (Foreman-Mackey et al. 2013), a Bayesian Markov chain Monte Carlo (MCMC) routine, wrapped around PHOEBE (Prša \& Zwitter 2005).

MCMC routines rely upon a Bayesian framework to evaluate the posterior probability of a given parameter set, given a data set, assuming a model. In our usage, the data set is the $K 2 \mathrm{LC}$ light curve, the model is produced by PHOEBE, and the parameter set is comprised of the mass ratio, inclination, semimajor axis (sma), third light, period and reference date of the system, as well as the potential and passband luminosity of either object and the effective temperature, spin/orbit synchronization and albedo of the secondary. Our MCMC routine evaluates 128 parameter chains for 5000 evaluations to produce 640000 model evaluations. Initially, the values for each parameter in a chain are drawn from uniform distributions with boundaries set as in Table 1. At each iteration, each parameter chain is used to construct a model using PHOEBE, which is then subject to a likelihood evaluation using the following form:

$\ln \mathcal{L}=-\frac{1}{2}\left(\frac{Y_{i}-M_{\Theta}}{\sigma_{i}}\right)=-\frac{1}{2} \chi^{2}$,

where $Y_{i}$ are the data, $\sigma_{i}$ are their uncertainties and $M_{\Theta}$ is the model produced with parameters $\Theta$.

PHOEBE allows for the direct interpolation of limb-darkening coefficients from pre-computed tables. Thus, we select the square-root limb-darkening law and interpolate at each iteration. Furthermore, drawing from the tables of Claret \& Bloemen (2011), we set the primary gravity darkening coefficient as $\beta_{1}=0.4007$, assuming $T_{\text {eff, } 1}=33000 \mathrm{~K}$ and $\log g_{1}=4.0 \mathrm{dex}$ (Mayer et al. 2013), such that the values adhere to the nearest values in the tables.

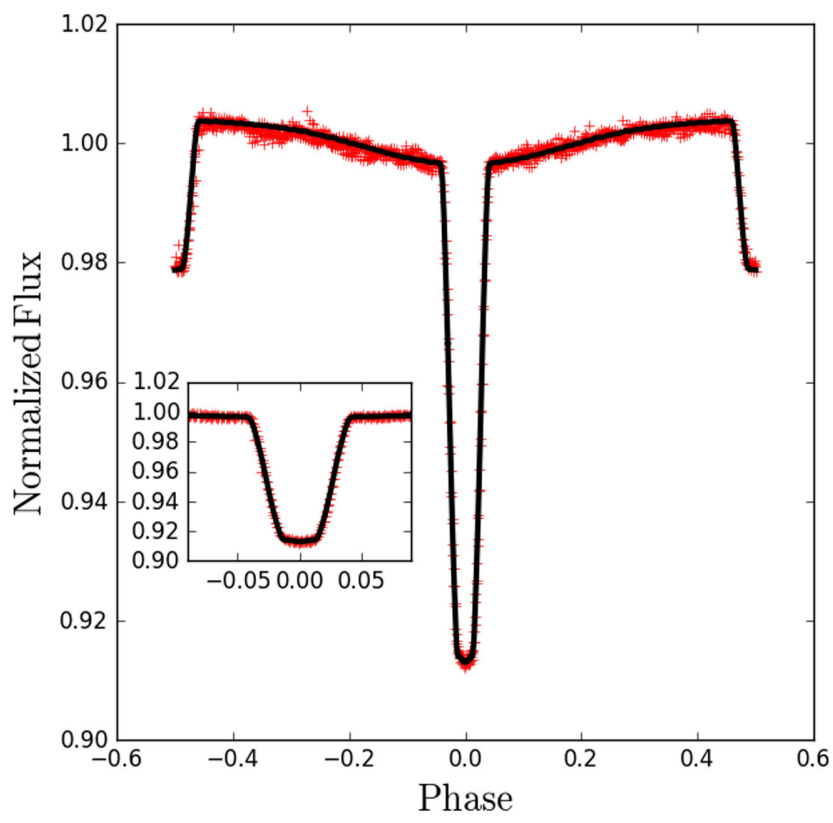

Figure 2. Phase-folded light curve of HD 165246 in red, with the binary model in black; the inset shows the zoomed-in view of the primary eclipse.

The MCMC algorithm converged well within the 5000 iterations, producing Gaussian posterior distributions for all parameters except sma and $A_{2}$. Final parameter estimates and accompanying uncertainties were drawn from the means and standard deviations of the posterior distributions of each parameter, as listed in Table 1. The final model is shown in Fig. 2. In contrast to Mayer et al. (2013), we do find a non-zero contribution of the third light $\left(L_{1}=80.2 \%, L_{2}=\right.$ $1.8 \%$ and $L_{3}=8 \%$ ) with an eccentricity of 0 , after we failed to converge to a non-zero eccentricity. The cause of the third light cannot be unravelled due to the large size of the Kepler pixels. Mayer et al. (2013) point out that, given the near $90^{\circ}$ argument of periastron, the eccentricity that they find might be spurious. Further long-term monitoring is required to determine whether the eccentricity they found is real or if the orbit is circular as we find.

Strong correlations occur between the mass ratio, $q$, and both potentials, $\Omega_{1}$ and $\Omega_{2}$; the primary passband luminosity, $L_{1}$, and third light, $L_{3}$; as well as the effective temperature and the albedo of the secondary, $T_{\text {eff, } 2}$ and $A_{2}$. The posteriors for $A_{2}$ optimized to 1 , the prior boundary we set, suggesting that the value be fixed at 1. Thus we did so, and repeated our modelling varying only $T_{\text {eff, }}$, removing the correlation in our results. The degeneracy between the mass ratio and potentials cannot be avoided without further spectroscopic observations. The posteriors for the sma are nearly uniform, suggesting that there is no information to constrain the value (within the prior range) in the data. We return to this in Section 4 to obtain reliable estimates for the primary and secondary masses.

\subsection{Intrinsic variability}

A final binary model was constructed from the values reported in Table 1 and removed from the light curve, resulting in the top panel of Fig. 3. A Lomb-Scargle periodogram of the residuals up to the Nyquist frequency of the LC data $\left(24.47 \mathrm{~d}^{-1}\right)$ reveals numerous frequency peaks (bottom panel of Fig. 3). The binary model was constructed for and subtracted from the SC light curve as well to confirm that no additional frequencies occur beyond $24.47 \mathrm{~d}^{-1}$. 

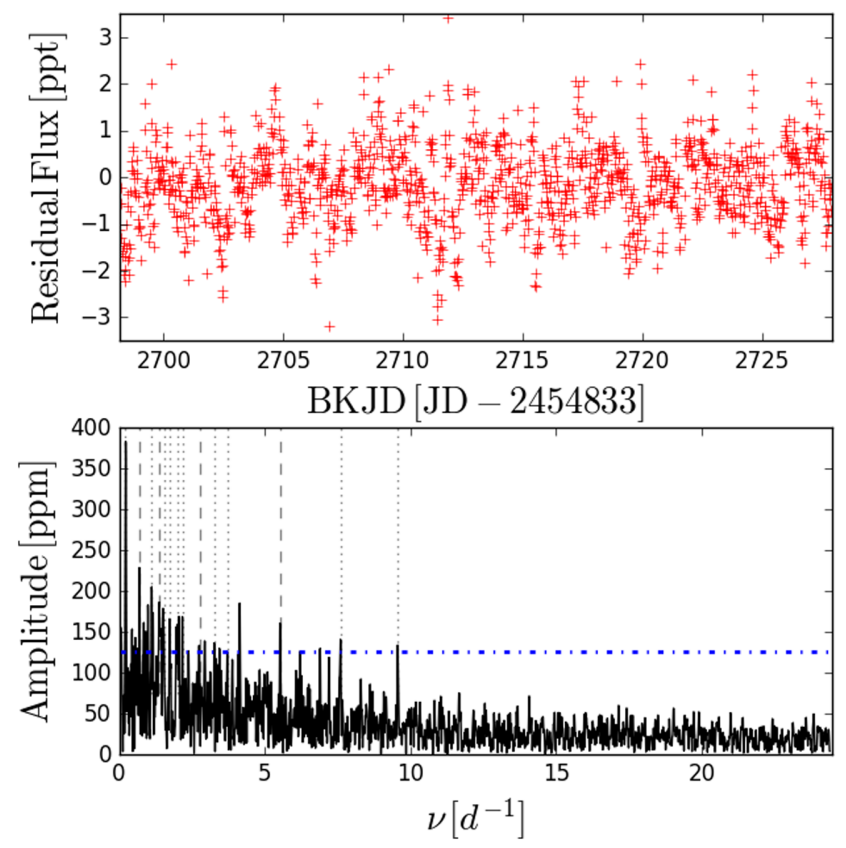

Figure 3. Top: residual light curve after removal of the binary model (Fig. 2). Bottom: Lomb-Scargle periodogram of the residual light curve. Dotted vertical lines indicate multiples of the orbital frequency, while dashed lines denote multiples of the rotation frequency. The horizontal line is placed at six times the average noise level after pre-whitening all frequencies in Table 2.

Since it has a longer time base, we proceed with the LC light curve to derive the frequency content of the residuals by applying a classical pre-whitening: after identification of the dominant frequency at each stage of the pre-whitening, we determine the frequency, amplitude and phase using a non-linear least-squares optimization. Following Degroote (2010), we allow the frequency to vary within 1.5 times the Rayleigh limit, $R_{\mathrm{L}}=0.034 \mathrm{~d}^{-1}$, and do not consider frequencies differing less than $0.05 \mathrm{~d}^{-1}$ in the pre-whitening process. The resulting frequencies with the signal-to-noise ratio $(\mathrm{S} / \mathrm{N}$; computed over [0, 24.47] $\mathrm{d}^{-1}$ ) above 6 after pre-whitening are reported in Table 2, along with their amplitudes, phases and their formal statistical errors. The latter are typically a factor 10 smaller than the frequency resolution, so we rather adopt $R_{\mathrm{L}}$ as a conservative more realistic error for the frequencies. In this way, we identify several frequencies as harmonics of the orbital frequency (as marked in Table 2), likely due to the imperfect removal of the binary signature. An asymmetry is observed between the out-of-eclipse light curve approaching and receding from the primary eclipse, possibly indicative of Doppler boosting (Bloemen et al. 2012) and could also explain the remaining harmonics seen in the periodogram. Furthermore, the frequencies $f=2.0544 \mathrm{~d}^{-1}$ and $f=4.1409 \mathrm{~d}^{-1}$ occur in the spectral window and are known to result from the semiperiodic thruster fires of the spacecraft.

From the projected orbital velocity of the primary in the case of synchronous rotation and the $v \sin i$ obtained from spectroscopy, Mayer et al. (2013) asserted that the synchronicity parameter of the primary is 3 . Adopting this, we identify $f=0.6967 \mathrm{~d}^{-1}$ as the rotation frequency. We observe a series of even harmonics of this frequency with decreasing amplitudes (Table 2). This is typical for a rotational signal intertwined with a gravity-mode oscillation spectrum in space photometry (e.g. Thoul et al. 2013). Indeed, we interpret the remaining independent significant frequencies in
Table 2. Extracted frequencies with formal statistical errors from non-linear least-squares fitting are given. The $\mathrm{S} / \mathrm{N}$ is computed over $[0,24.47] \mathrm{d}^{-1}$ after pre-whitening. Two instrumental frequencies occur.

\begin{tabular}{|c|c|c|c|c|}
\hline $\begin{array}{l}\text { Frequency } \\
\left(\mathrm{d}^{-1}\right)\end{array}$ & $\begin{array}{l}\text { Amplitude } \\
\text { (ppm) }\end{array}$ & $\begin{array}{r}\text { Phase } \\
\text { modulo } 2 \pi\end{array}$ & $\mathrm{S} / \mathrm{N}$ & Remarks \\
\hline $0.0659 \pm 0.0036$ & $168 \pm 32$ & $-0.39 \pm 0.07$ & 8.02 & \\
\hline $0.2180 \pm 0.0025$ & $259 \pm 35$ & $-0.03 \pm 0.04$ & 12.34 & forb \\
\hline $0.2698 \pm 0.0028$ & $251 \pm 39$ & $-0.38 \pm 0.05$ & 11.97 & \\
\hline $0.4956 \pm 0.0041$ & $131 \pm 28$ & $0.15 \pm 0.07$ & 6.23 & \\
\hline $0.6165 \pm 0.0038$ & $135 \pm 28$ & $0.28 \pm 0.07$ & 6.43 & \\
\hline $0.6967 \pm 0.0027$ & $241 \pm 35$ & $-0.13 \pm 0.05$ & 11.49 & frot \\
\hline $0.9945 \pm 0.0037$ & $152 \pm 31$ & $0.45 \pm 0.06$ & 7.26 & \\
\hline $1.1205 \pm 0.0031$ & $202 \pm 34$ & $-0.25 \pm 0.05$ & 9.61 & 5 forb \\
\hline $1.3841 \pm 0.0034$ & $179 \pm 33$ & $0.33 \pm 0.06$ & 8.52 & 2 frot \\
\hline $1.4247 \pm 0.0038$ & $146 \pm 30$ & $-0.32 \pm 0.07$ & 6.96 & \\
\hline $1.5090 \pm 0.0031$ & $193 \pm 33$ & $0.38 \pm 0.05$ & 9.17 & \\
\hline $1.5434 \pm 0.0037$ & $147 \pm 29$ & $0.13 \pm 0.06$ & 6.99 & 7 forb \\
\hline $1.7360 \pm 0.0037$ & $157 \pm 31$ & $0.41 \pm 0.06$ & 7.47 & 8 forb \\
\hline $1.9562 \pm 0.0040$ & $135 \pm 29$ & $-0.38 \pm 0.07$ & 6.43 & \\
\hline $2.0077 \pm 0.0036$ & $158 \pm 31$ & $0.50 \pm 0.06$ & 7.51 & 9 forb \\
\hline $2.0544 \pm 0.0038$ & $146 \pm 30$ & $0.02 \pm 0.07$ & 6.98 & inst \\
\hline $2.1706 \pm 0.0033$ & $182 \pm 32$ & $-0.19 \pm 0.06$ & 8.68 & 10 forb \\
\hline $2.7544 \pm 0.0038$ & $133 \pm 28$ & $0.21 \pm 0.07$ & 6.34 & 4 frot \\
\hline $2.9471 \pm 0.0040$ & $129 \pm 28$ & $0.13 \pm 0.07$ & 6.13 & \\
\hline $3.2692 \pm 0.0040$ & $133 \pm 29$ & $-0.12 \pm 0.07$ & 6.36 & 15 forb \\
\hline $3.7126 \pm 0.0039$ & $127 \pm 27$ & $0.24 \pm 0.07$ & 6.06 & 17 forb \\
\hline $4.1400 \pm 0.0033$ & $187 \pm 34$ & $-0.20 \pm 0.06$ & 8.93 & inst \\
\hline $5.5249 \pm 0.0035$ & $165 \pm 32$ & $-0.43 \pm 0.06$ & 7.88 & 8 frot \\
\hline $7.5975 \pm 0.0038$ & $145 \pm 30$ & $0.03 \pm 0.07$ & 6.91 & 35 forb \\
\hline $9.5630 \pm 0.0039$ & $135 \pm 28$ & $-0.09 \pm 0.07$ & 6.42 & 44 forb \\
\hline
\end{tabular}

Table 2 as caused by oscillations. They can be due to heat-driven coherent modes resulting in isolated eigenfrequencies (Briquet et al. 2011) or rather be connected with a whole spectrum of frequencies as detected in three other $\mathrm{O}$ stars observed with the COROT space mission (Blomme et al. 2011) and interpreted as due to IGW (Aerts \& Rogers 2015) or a combination thereof. The limited frequency resolution does not allow us to distinguish among these phenomena nor to detect frequency or period spacing patterns. Nevertheless, we conclude that the HD 165246 system exhibits intrinsic oscillatory variability with amplitudes of $\sim 150 \mathrm{ppm}$ level and could provide a benchmark for $\mathrm{O}$-star physics using complementary binary and asteroseismic modelling.

\section{CONSTRAINING STELLAR MODELS}

Using the reported effective temperature of the primary and derived parameters from PHOEBE, we build a set of stellar models with the MESA stellar evolution code (Paxton et al. 2015, and references therein, V8118) with the sole aim to constrain the masses of the binary components, while detailed modelling will be the subject of a follow-up paper based on new spectroscopy to be gathered. Our grid consists of models with masses $16 \mathrm{M}_{\odot} \leq \mathrm{M} \leq 25 \mathrm{M}_{\odot}$ in steps of $0.5 \mathrm{M}_{\odot}$, adopting solar metallicity, an exponential overshooting with a parameter of $f_{\mathrm{ov}}=0.02$ and mixing length parameter $\alpha_{\mathrm{mlt}}=$ 2.0. We apply a mass loss due to a radiation-driven wind using an efficiency parameter $\eta_{\text {Vink }}=0.5$ in MESA (Vink, de Koter \& Lamers 2001).

Models with an initial mass below $18.5 \mathrm{M}_{\odot}$ never reach the $3 \sigma$ effective temperature range reported by Mayer et al. (2013) and evolve to lower temperatures than $32500 \mathrm{~K}$ after $1.23 \mathrm{Myr}$, leading us to dispose those. This also serves as a lower limit for the 
age of the system. Given the uncertainties on the mass ratio determined through our binary modelling, we can eliminate values of the semimajor axis below sma $=32.7 \mathrm{R} \odot$. Models with initial masses up to, and including, $25 \mathrm{M}_{\odot}$ cross the regions where the effective temperature is consistent with spectroscopy. This produces a final mass range of $19 \mathrm{M}_{\odot} \leq \mathrm{M}_{1} \leq 25 \mathrm{M}_{\odot}$ for the primary and a range of $32.7 \mathrm{R}_{\odot} \leq \mathrm{sma} \leq 36 \mathrm{R}_{\odot}$ for the semimajor axis. Given the mass ratio obtained from binary modelling, we find a mass range of $3.3 \mathrm{M}_{\odot} \leq \mathrm{M}_{2} \leq 4.4 \mathrm{M}_{\odot}$ for the secondary. While the evolutionary tracks for these masses pass through the corresponding $3 \sigma$ effective temperature found with PHOEBE, the $\log g$ is entirely unconstrained by the spectroscopy from the Mayer et al. (2013) analysis and is instead assumed from their PHOEBE model. Due to degeneracies present in the binary modelling procedure, the determination of the surface gravities of the components from new high-S/N spectroscopy is needed to break these degeneracies and further constrain theoretical models of the system, with the potential of seismic calibration for high-mass stellar models. These types of data are also needed to exclude that (some of) the detected frequencies listed in Table 2 are due to the secondary or other components.

Indeed, our mass estimate of the secondary places it in the instability strip of slowly pulsating B stars, where gravity modes are expected (Moravveji 2016). Although these have a narrower frequency range and a different morphology in Fourier space than what we observe for HD 165246 (see fig. 10 in Pápics et al. 2017), we cannot exclude that some of the detected frequencies are due to the secondary's 1.8 per cent or the composite 18 per cent third light contribution to the flux, as oscillation modes of amplitude above $10 \mathrm{ppt}$ do occur in B-type pulsators. However, given reported amplitudes for IGWs in recent literature (3000 ppm; Aerts \& Rogers 2015; Aerts et al. 2017), a contaminating source would need to contribute $\sim 50$ per cent light to the system to produce the observed amplitude and would thus be seen in the spectra. Therefore, the IGW signal can only be produced by the hot O-type primary.

\section{CONCLUSIONS}

This work has identified HD 165246 as the second massive O+B binary with signatures of rotational and seismic frequencies in highprecision space photometry. Using 29.77 d of $K 2$ data and relying on spectroscopy in the literature, we achieved a binary model of the system. We further detected variability in the residuals after subtraction of the binary model and identified the rotational frequency of the O-type primary in the $K 2$ data. The orbital frequency and several of its harmonics are still present in the residuals, suggesting missing physics in the binary modelling and/or additional binary variability not yet taken into account in our model. Aside from binary and rotational variability, we detected additional frequencies pointing towards seismic variability. The current $K 2$ time series is too short to allow for an unambiguous interpretation in terms of coherent oscillation modes or IGWs.

Given the high potential of HD 165246 to perform binary and seismic modelling, but the low amplitude of the photometric variability, we will embark on long-term high-precision time-series spectroscopy with various instruments and covering several orbital periods, with the aim to clarify the nature of the secondary and tertiary components, as well as to detect and interpret the variability from dedicated spectral line diagnostics. Only then can we perform a full seismic modelling of this multiple system.

\section{ACKNOWLEDGEMENTS}

The research leading to these results has received funding from the European Research Council (ERC) under the European Union's Horizon 2020 research and innovation programme (grant agreement no. 670519: MAMSIE). The research presented here makes use of the MAST archive hosted by STScI in the USA and the Simbad astronomical database hosted in CDS, Stroudsbourg, France. This paper includes data collected by the Kepler mission. Funding for the Kepler mission is provided by the NASA Science Mission directorate.

\section{REFERENCES}

Aerts C., Rogers T. M., 2015, ApJ, 806, L33

Aerts C. et al., 2017, A\&A, in press, preprint (arXiv:1703.01514)

Alvan L., Strugarek A., Brun A. S., Mathis S., Garcia R. A., 2015, A\&A, 581, A112

Auvergne M. et al., 2009, A\&A, 506, 411

Bloemen S. et al., 2012, MNRAS, 422, 2600

Blomme R. et al., 2011, A\&A, 533, A4

Briquet M. et al., 2011, A\&A, 527, A112

Buysschaert B. et al., 2015, MNRAS, 453, 89

Claret A., Bloemen S., 2011, A\&A, 529, A75

Claret A., Torres G., 2016, A\&A, 592, A15

Degroote P., 2010, PhD thesis, KU Leuven, Belgium

Foreman-Mackey D., Hogg D. W., Lang D., Goodman J., 2013, PASP, 125, 306

Hambleton K. et al., 2016, MNRAS, 463, 1199

Howell S. B. et al., 2014, PASP, 126, 398

Koch D. G. et al., 2010, ApJ, 713, L79

Kurtz D. W., Saio H., Takata M., Shibahashi H., Murphy S. J., Sekii T., 2014, MNRAS, 444, 102

Mayer P., Harmanec P., Pavlovski K., 2013, A\&A, 550, A2

Moravveji E., 2016, MNRAS, 455, L67

Moravveji E., Townsend R. H. D., Aerts C., Mathis S., 2016, ApJ, 823, 130

Murphy S. J., Fossati L., Bedding T. R., Saio H., Kurtz D. W., Grassitelli L., Wang E. S., 2016, MNRAS, 459, 1201

Otero S., 2007, Open Eur. J. Var. Star, 72, 1

Pablo H. et al., 2017, MNRAS, 467, 2494

Pápics P. I. et al., 2017, A\&A, 598, A74

Paxton B. et al., 2015, ApJS, 220, 15

Prša A., Zwitter T., 2005, ApJ, 628, 426

Rogers T. M., Lin D. N. C., McElwaine J. N., Lau H. H. B., 2013, ApJ, 772 , 21

Saio H., Kurtz D. W., Takata M., Shibahashi H., Murphy S. J., Sekii T., Bedding T. R., 2015, MNRAS, 447, 3264

Sana H. et al., 2014, ApJS, 215, 15

Schmid V. S., Aerts C., 2016, A\&A, 592, A116

Thoul A. et al., 2013, A\&A, 551, A12

Triana S. A., Moravveji E., Pápics P. I., Aerts C., Kawaler S. D., ChristensenDalsgaard J., 2015, ApJ, 810, 16

Van Reeth T. et al., 2015, ApJS, 218, 27

Van Reeth T., Tkachenko A., Aerts C., 2016, A\&A, 593, A120

Vanderburg A., Johnson J. A., 2014, PASP, 126, 948

Vink J. S., de Koter A., Lamers H. J. G. L. M., 2001, A\&A, 369, 574

Walborn N. R., 1972, AJ, 77, 312

Welsh W. F. et al., 2011, ApJS, 197, 4

This paper has been typeset from a $\mathrm{T}_{\mathrm{E}} \mathrm{X} / \mathrm{LAT}_{\mathrm{E}} \mathrm{X}$ file prepared by the author. 